\title{
Transalpina
}

TRANSALPINA Études italiennes

\section{3 | 2020}

Enseigner l'italien en Langues Étrangères Appliquées

\section{Mario Lavagetto, «Oltre le usate leggi ». Una lettura del Decameron}

\section{Luca Bani}

\section{(2) OpenEdition}

1 Journals

\section{Edizione digitale}

URL: https://journals.openedition.org/transalpina/723

DOI: 10.4000/transalpina.723

ISSN: 2534-5184

\section{Editore}

Presses universitaires de Caen

\section{Edizione cartacea}

Data di pubblicazione: 1 novembre 2020

Paginazione: 181-183

ISBN: 978-2-84133-989-1

ISSN: $1278-334 \mathrm{X}$

Notizia bibliografica digitale

Luca Bani, «Mario Lavagetto, «Oltre le usate leggi ». Una lettura del Decameron», Transalpina [Online], 23 |

2020, online dal 01 novembre 2020, consultato il 21 octobre 2022. URL: http://

journals.openedition.org/transalpina/723 ; DOI: https://doi.org/10.4000/transalpina.723

All rights reserved 


\title{
NOTES CRITIQUES
}

\author{
Mario Lavagetto, «Oltre le usate leggi». Una lettura del Decameron, \\ Torino, Einaudi, 2019, 256 p.
}

Mario Lavagetto, almeno per quanto riguarda le sue opere maggiori, ci aveva abituato a una critica rivolta agli autori e ai fenomeni letterari legati al contemporaneo: Saba, Svevo, Proust e Freud sono i suoi riconosciuti campi di indagine, accompagnati da ricerche sempre su autori del Novecento che si potrebbero solo impropriamente definire collaterali (Calvino), da piacevoli escursioni nei territori ottocenteschi (i libretti verdiani, Balzac) e da sguardi più trasversali legati alla persistenza nel tempo di particolari espedienti narrativi (La cicatrice di Montaigne). A nostra memoria, l'Autore aveva superato i confini cronologici del campo di ricerca privilegiato solo collaborando a una miscellanea del 1982 (Il testo moltiplicato. Letture di una novella del Decameron) della quale assunse anche la curatela, ma questo, ovviamente, non significa che nei suoi corsi non li avesse mai travalicati, e "Oltre le usate leggi» ne è la prova. Infatti, il testo si fonda su un ciclo di lezioni tenute all'Università di Basilea che Lavagetto ha sottoposto a un lungo lavoro di rielaborazione, affidandosi metodologicamente agli strumenti messi a disposizione dalla teoria della letteratura, usati però con prudenza e leggerezza, per scandagliare alcune novelle ritenute più rappresentative e con l'intento di "vedere se era possibile mettere in salvo il Decameron dalle sue "traduzioni" " (p. XI), ovvero, nello spirito della più pura filologia, di riportare la parola boccacciana al suo dettato originario, purificandola dalle traduzioni, riscritture e interpretazioni (Branca in particolare), che secondo l'Autore nel corso dei secoli ne hanno inquinato o anche solo deviato il senso, il timbro, la filatura e il colore. In una parola, l'intento di Lavagetto è non solo quello di restituire leggibilità al testo, ma di renderlo anche amico del lettore, per smentire la previsione fatta da Paul Valéry nel 1923, e ricordata nella Premessa, sull'imminente incapacità delle nuove generazioni di comprendere i classici. Questa è la scommessa.

Strutturato in sette capitoli (Galeotto, La cornice, Ascoltare Raccontare, Tre donne, Mentire, Figure della «bêtise » e Quale Griselda) e un'Appendice, il volume si apre con la sostanziale riaffermazione del valore delle tematiche sessuali nel Decameron, della rilevanza che in esso è attribuito al corpo e dell'importanza che Boccaccio dà alla dimensione dell'incontro fisico tra gli esseri umani, il tutto esplicitamente dichiarato nell' incipit con l'attribuzione 
all'opera di un significativo cognome: Galeotto, ossia intermediario di quegli incontri intesi a soddisfare i desideri carnali, spirituali o entrambi degli amanti. Ma una così aperta affermazione della felicità legata alla soddisfazione della voce del corpo e, indirettamente, del legame esistente tra corpo e spirito, non poteva essere accettata in una società e in una cultura ancora così fortemente intrise di idiosincrasie sessuali. Ecco quindi la condanna di Petrarca, l'abiura stessa che Boccaccio fece della sua opera, per arrivare nel Cinquecento alla messa all'Indice (1558) e alle conseguenti riscritture del testo (Vincenzo Borghini nel 1573, Leonardo Salviati nel 1588, Luigi Groto sempre nel 1588), più o meno profonde, ma tutte rivolte a mondare le parti più pruriginose del testo, e fra di esse soprattutto quelle che vedevano protagonisti membri maschili o femminili della Chiesa.

Anche per quanto riguarda la cornice dell'opera, Lavagetto punta a far piazza pulita di tutte quelle interpretazioni che sono andate oltre la lettera del testo attribuendo a questo elemento strutturale significati che «spingono idealisticamente in primo piano la reattività e le sensazioni del singolo lettore» (p. 58), e non è un caso che subito dopo venga citato Croce, e analizzando invece il ruolo che essa assume nel complesso rapporto tra la narrazione autoriale e le voci narranti delle sette giovani e dei loro tre compagni, ossia nella dinamica tra le cose viste direttamente da Boccaccio e le cose udite da un testimone oculare (una voce femminile?) che ripensa il ruolo dell'Autore e quello della cornice come confine tra una sua effettiva esperienza diretta, la peste, e una serie di esperienze riportate, le novelle.

Ma il vero centro della lettura e dell'analisi di Lavagetto sono le donne, portatrici principali di quell'intelligenza limpidamente beffarda che si irradia per tutte le dieci giornate e che con intuizione originale, e con tutta probabilità effettivamente anticipatrice del desiderio umanistico di conoscere ed esplorare l'intima natura dell'essere umano, contribuisce a delineare la complessità dell'animo, della psicologia e dei desideri femminili. A queste figure Lavagetto, così come Boccaccio, dedica le pagine più intense del suo scritto: le riflessioni sulle narratrici, Elissa, Filomena e le altre, e sulle protagoniste delle vicende narrate, Bartolomea Gualandi (II, 10), Ghismunda (IV, 1), Madonna Filippa (VI, 7) e Griselda (V, 10) vengono introdotte dall'Autore con un'elencazione di definizioni morali, fisiche e caratteriali ripresa da Amedeo Quondam (Le cose (e le parole) del mondo, in Boccaccio, Decameron, Milano, BUR, 2013, p. 1690 e sg.) e che risuona più come una dichiarazione d'amore che come un'algida premessa critica: "Vaghe, dilicate, graziose, graziosissime, valorose oneste, amorose, giovani, care, carissime, religiose, tenere, belle, bellissime, piacevoli, discrete, dolcissime, pietose, avvedute, nobili, nobilissime, reverende, dilettose, onestissime, gentilissime, laudevoli, soavissime, amabili, leggiadre, innamorate, 
labili, inchinevoli, adorne, onorabili, benigne, morbide, moderate, discretissime, amorevoli, splendide, ragguardevoli, magnifiche: le donne del Decameron» (p. 103).

Nessuna delle donne di Boccaccio passa inosservata o resta confinata nella diafana e spersonalizzante caratterizzazione nella quale sono state generalmente rinchiuse dalla casistica medievale, tranne alcune lodevoli e significative eccezioni, ma ciascuna mette in luce un tratto particolare della personalità femminile che, insieme agli altri, consente al lettore attento di affacciarsi su un orizzonte del tutto nuovo e inusuale per l'epoca.

Attraverso l'evidenza data a queste figure e l'applicazione di una metodologia non soverchiante, ma utile a valorizzare le prospettive di volta in volta dichiarate dallo studioso, Lavagetto restituisce al testo l'integrità del messaggio primigenio e la vividezza della voce con cui questo viene trasmesso al lettore; e lo fa richiamando, nelle pagine finali dell'Appendice, il magistero di De Sanctis, di Debenedetti e di Bachtin, e quindi rifacendosi a una tradizione esegetica che riporta l'eterodossia dichiarata del suo sguardo nell'alveo di una ben radicata ortodossia interpretativa.

Luca BANI

\section{Massimiliano Biscuso, Leopardi tra i filosofi. Spinoza, Vico, Kant, Nietzsche, Napoli, La Scuola di Pitagora, 2019, 223 p.}

Massimiliano Biscuso è membro dell'Istituto Italiano per gli Studi Filosofici, presso il quale oltre ad avere responsabilità gestionali - fa parte del Consiglio esecutivo - svolge attività didattica e di ricerca. Per quest'ultima si è occupato prevalentemente di problemi e momenti di storia della filosofia ottocentesca e di filosofi di questo secolo. Molto ha scritto anche su Leopardi, che evidentemente rappresenta uno dei suoi principali oggetti di riflessione e di studio: Leopardi antitaliano (con Franco Gallo, Manifesto Libri, 1999), Oltre il nichilismo, Leopardi (Edizioni Scientifiche Italiane, 2009), Gli usi di Leopardi : figure del leopardismo filosofico italiano (Manifesto Libri, 2019) $\mathrm{e}$, infine, il volume a cui è dedicata questa recensione. Una tetralogia, dunque, che trova in quest'ultimo volume una sorta di sintesi tesa a collocare definitivamente Leopardi nel posto che gli compete nel pantheon della filosofia europea, «filosofo tra gli altri filosofi» (p. 9), sia collegandolo con la tradizione precedente - Spinoza, Vico e Kant, appunto - sia approfondendo lo sguardo su una delle più intriganti ricezioni postume del Recanatese, quella nietzschiana.

Che Leopardi, insieme a Dante, sia per la letteratura italiana il più alto rappresentante del possibile e felice connubio tra pensiero filosofico ed 